\title{
Commentary: Infective endocarditis: Finding the right time for the right side
}

\author{
Jason J. Han, MD, and Pavan Atluri, MD
}

\footnotetext{
From the Division of Cardiovascular Surgery, Department of Surgery, University of Pennsylvania, Philadelphia, $\mathrm{Pa}$.

Disclosures: Authors have nothing to disclose with regard to commercial support.

Received for publication Aug 7, 2018; accepted for publication Aug 10, 2018; available ahead of print Sept 20, 2018.

Address for reprints: Pavan Atluri, MD, Division of Cardiovascular Surgery, Hospital of the University of Pennsylvania, 3400 Spruce St, 6 Silverstein Pavilion, Philadelphia, PA 19104 (E-mail: Pavan.Atluri@uphs.upenn. edu).

J Thorac Cardiovasc Surg 2019;157:1428-9

$0022-5223 / \$ 36.00$

Copyright (c) 2018 by The American Association for Thoracic Surgery

https://doi.org/10.1016/j.jtcvs.2018.08.026
}

Surgical management of right-sided infective endocarditis is a multifaceted challenge clinically and in research. It is relatively uncommon, comprising only $5 \%$ to $10 \%$ of all infective endocarditis cases, of which only $15 \%$ to $20 \%$ of these require surgery. ${ }^{1,2}$ The disease profile is also heterogeneous. The various permutations of etiologies, causative organisms and affected structures render a standardized approach to its treatment elusive. In this context, the contribution by Witten and colleagues ${ }^{3}$ in this issue of the Journal is appropriate and timely. As they note, most studies to date have only focused on a single aspect of the issue, ${ }^{4}$ most notably its relation to intravenous drug use (IVDU). ${ }^{5}$ This has been appropriate, given the complexity and growing scale of the issue. Posing both a medical and a social challenge, the use of heroin in the United States nearly doubled between 2006 and 2013, ${ }^{6}$ with a commensurate spike in the incidence of right-sided infective endocarditis.

One the strengths of this data set is its ability to present a balanced comparison of multiple predisposing conditions, such as cardiac implantable devices and chronic vascular access, in addition to IVDU. These cohort stratifications may form a precedent in informing similar efforts at other hospitals better to understand and thereby treat this patient population. In many ways, the article confirms what we already know, such as the predominance of Staphylococcus aureus among patients with a history of IVDU, ${ }^{7}$ and the survival benefit observed among patients who receive valve repair or reconstruction rather than replacement. ${ }^{8,9}$ Yet it also sheds insight on relatively nebulous areas. such as surgical indications for right-sided infective endocarditis, which have not been as clearly delineated as those for left-sided infective endocarditis. Not surprisingly, risk of septic embolism significantly added urgency to operating on patients with a history of IVDU to prevent pulmonary complications. Chronic vascular access patients had the lowest early and late-term survivals, likely because of worse health at baseline. Recurrence rate was minimal for patients

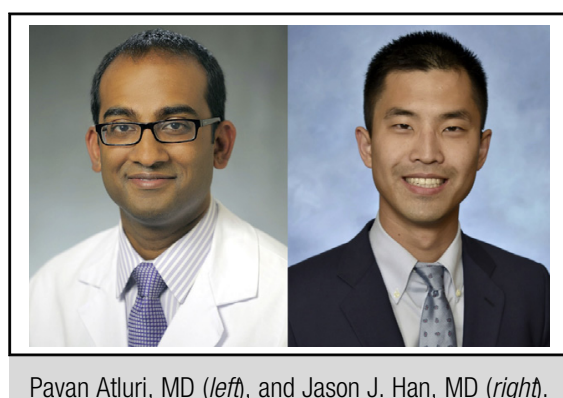

\section{Central Message \\ Right-sided infective endocarditis poses a multifaceted challenged both clinically and in research. This article thoroughly characterizes its heterogeneous patient population.}

See Article page 1418 . with non-IVDU indications. Although the heterogeneity of the population added a challenge to its extrapolation, Witten and colleagues ${ }^{3}$ have conducted extensive, supplemental statistical analysis to buttress these findings. Inevitably, there is an inherent selection bias in retrospectively defining a cohort that has been referred specifically to receive operations at a quaternary center; its findings thus are limited to only the surgically managed subset of patients.

Overall, Witten and colleagues ${ }^{3}$ are to be congratulated for sharing their impressive institutional experience and detailed statistical analysis. Moreover, their strong emphasis on a multidisciplinary team approach, which "reflects a culture existing ahead of guidelines," is undeniably a factor behind their excellent outcomes and gives us a reason to be optimistic that this challenging medical, surgical, and social issue will continue to be better managed in the future.

\section{References}

1. Prendergast BD, Tornos P. Surgery for infective endocarditis: who and when? Circulation. 2010;121:1141-52.

2. Akinosoglou K, Apostolakis E, Koutsogiannis N, Leivaditis V, Gogos CA. Rightsided infective endocarditis: surgical management. Eur J Cardiothorac Surg. 2012;42:470-9.

3. Witten JC, Hussain ST, Shrestha NK, Gordon SM, Houghtaling PL, Bakaeen FG, et al. Surgical treatment of right-sided infective endocarditis. J Thorac Cardiovasc Surg. 2019;157:1418-27.e14.

4. Malekzadeh-Milani S, Ladouceur M, Iserin L, Bonnet D, Boudjemline Y. Incidence and outcomes of right-sided endocarditis in patients with congenital heart disease after surgical or transcatheter pulmonary valve implantation. J Thorac Cardiovasc Surg. 2014;148:2253-9. 
5. Arbulu A, Holmes RJ, Asfaw I. Surgical treatment of intractable right-sided infective endocarditis in drug addicts: 25 years experience. J Heart Valve Dis. 1993;2: 129-37; discussion 138-9.

6. Substance Abuse and Mental Health Services Administration Center for Behavioral Health Statistics and Quality. Results from the 2013 National Survey on Drug Use and Health: summary of national findings. Available at: https://www. samhsa.gov/data/sites/default/files/NSDUHresultsPDFWHTML2013/Web/NSDUH results2013.pdf.
7. Right-sided endocarditis with Staphylococcus aureus. Ann Intern Med. 1989;110 496-8.

8. Jiang SL, Li BJ, Zhang T, Ren CL, Wang Y, Chen TT, et al. Surgical treatment of isolated right-sided infective endocarditis. Tex Heart Inst J. 2011;38:639-42.

9. Musci M, Siniawski H, Pasic M, Grauhan O, Weng Y, Meyer R, et al. Surgical treatment of right-sided active infective endocarditis with or without involvement of the left heart: 20-year single center experience. Eur J Cardiothorac Surg. 2007; $32: 118-25$ 